Pacific Journal of Mathematics

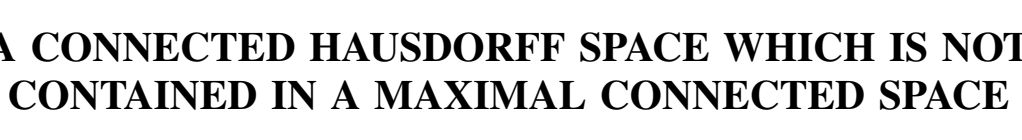




\title{
A CONNECTED HAUSDORFF SPACE WHICH IS NOT CONTAINED IN A MAXIMAL CONNECTED SPACE
}

\author{
IVAN BAGGS
}

\begin{abstract}
An example of a countable connected Hausdorff space $(X, \sigma)$ is given which has the property that for every topology $\gamma$ strictly larger than $\sigma$, where $(X, \gamma)$ is connected, there exists a topology $\gamma^{\prime}$, strictly larger than $\gamma$, such that $\left(X, \gamma^{\prime}\right)$ is connected. There also exists uncountable connected Hausdorff spaces which have this property.
\end{abstract}

The purpose of this note is to present an example of a connected Hausdorff space which cannot be embedded into a maximal connected space.

1. Preliminaries. $R$ will be used to denote the real line with the usual topology, $\tau$. Let $X$ be a set and let $A$ and $B$ be families of subsets of $X$, then $A \vee B$ denotes the topology generated by the subbase $P=\{C \mid C \in A$ or $C \in B\}$.

DEFINITION 1 (see [4]). A connected topological space $(X, \tau)$ is maximal connected if for every topology $\tau_{1}$, where $\tau_{1}$ is strictly larger than $\tau,\left(X, \tau_{1}\right)$ is not connected.

REMARK 1. Let $X$ be a set and $\tau_{n}, n=1,2,3, \cdots$, be a sequence of topologies on $X$ such that $\left(X, \tau_{n}\right)$ is connected for $n=1,2, \cdots$. If $\tau$ is the topology which is the least upper bound of the sequence $\tau_{n}, n=1,2, \cdots$, then $(X, \tau)$ is not necessarily connected (see problem 5 , page $155,[1])$.

Definition 2. A topological space $(X, \tau)$ is maximal perfect if $(X, \tau)$ has no isolated points and, for every topology $\tau_{1}$ strictly larger than $\tau,\left(X, \tau_{1}\right)$ has an isolated point.

LEMMA 1. If $(X, \tau)$ is any topological space without isolated points, then there exists a topology $\tau_{1} \supset \tau$ such that $\left(X, \tau_{1}\right)$ is maximal perfect.

Proof. Let $\left\{\tau_{\alpha}\right\}_{\alpha \in A}$ be a linearly ordered family of topologies on $X$ such that, for each $\alpha \in A, \tau_{\alpha} \supset \tau$ and $\left(X, \tau_{\alpha}\right)$ has no isolated points. Let $\sigma$ be the topology generated by $\left\{\tau_{\alpha}\right\}_{\alpha \in A}$. If there exists some $x \in X$ such that $\{x\} \in \sigma$, then, since $\left\{\tau_{\alpha}\right\}_{\alpha \in A}$ is linearly ordered, $\{x\} \in \tau_{\alpha}$, for some $\alpha$. Therefore, $(X, \sigma)$ has no isolated points. It therefore 
follows by Zorn's lemma that there exists a topology $\tau_{1} \supset \tau$ such that $\left(X, \tau_{1}\right)$ is maximal perfect.

2. Particular maximal perfect topologies on sets of rationals. In constructing the topological space $(X, \sigma)$ referred to in the title, we will make use of the fact that the restriction of $\sigma$ to certain subsets of $X$ is maximal perfect. However, in order to make full use of this fact, we need to know something about the structure of the open subsets of these maximal perfect subspaces. Consequently, we now put a particular maximal perfect topology on a dense subspace of the rationals. We will make use of this model in constructing the required topological space.

Let $P$ denote a dense subset of the rationals with the topology $\tau$ inherited from $R$.

Definition 3. A set $G \subset P$ is an $N$-set if $G=\dot{\phi}$ or if for each $x \in G$ and for every $b>x,\{y \in G \mid x<y<b\}$ has nonempty $\tau$-interior.

Definition 4. A collection $\mathscr{G}$ of $N$-sets which is closed under finite intersections is an $N$-family.

It follows from Zorn's lemma that every $N$-family is contained in a maximal $N$-family. For each $x \in P$, let $I_{x}=\{y \in P \mid x \leqq y<x+1\}$. Put $B=\left\{I_{x} \mid x \in P\right\} \cup\{0 \subset P \mid 0 \in \tau\} . \quad B$ is contained in an $N$-family, $B_{1}$. Let $\mathscr{M}$ be a maximal $N$-family containing $B_{1}$.

If $D$ is a subset of $P$, then $D^{c}$ denotes the complement of $D$ in $P$. Let $Q=\left\{D \subset P \mid D^{c}\right.$ is $\tau$-nowhere dense in $\left.P\right\}$. Then $Q$ is contained in a filter $\mathscr{F}$, where $\mathscr{F}$ is maximal with respect to the properties that, (i) $Q \subset \mathscr{F}$ and (ii) if $F \in \mathscr{F}$, then $F$ is $\tau$-dense in $P$. Throughout, $\mathscr{C}$ and $\mathscr{F}$ will be used, respectively, to denote a maximal family of $\mathrm{N}$-sets and a maximal filter of dense sets constructed as outlined above.

Lemma 2. Let $\sigma=\mathscr{C} \vee \mathscr{F}$. Then $\tau \subset \sigma$ and $(P, \sigma)$ is a maximal perfect space.

Proof. Since $\tau \subset \mathscr{l}$, it follows that $\tau \subset \sigma$. Let $x \in P$ and let $x \in F \cap M$, where $F \in \mathscr{F}$ and $M \in \mathscr{L}$. Since $M$ contains a $\tau$-open set $U$ and $F \cap U$ is $\tau$-dense in $U$, it follows that $F \cap M \neq\{x\}$. Therefore, $(P, \sigma)$ is perfect.

Suppose $(P, \sigma)$ is not maximal perfect. Then there exists a topology $\gamma$ on $P$ such that $\gamma$ is strictly larger than $\sigma$ and $(P, \gamma)$ is perfect. Let $V \in \gamma$ such that $V \notin \sigma$. Let $x_{0}$ be an arbitrary fixed element of $V$. For every $b>x_{0},\left\{x \in P \mid x_{0}<x<b\right\} \cap V \neq \phi$. [Otherwise, for some $b>x_{0}, V \cap\left\{x \in P \mid x_{0}-1<x<b\right\} \cap I_{x_{0}}=\left\{x_{0}\right\}$, where $I_{x_{0}}=$ 
$\left\{x \in P \mid x_{0} \leqq x<x_{0}+1\right\}$, and $\left\{x_{0}\right\}$ would be an element of $\gamma$ which is impossible.]

Suppose there exists some $b>x_{0}$ such that $D=\left\{x \in P \mid x_{0}<x<b\right\} \cap V$ is $\tau$-nowhere dense in $\left\{x \in P \mid x_{0}<x<b\right\}$. Then $D \in \gamma$ and it follows from the construction of $\mathscr{F}$ that $D^{c} \in \mathscr{F}$. If $y$ is a fixed element of $D$, then $\left(D^{a} \cup\{y\}\right) \in \mathscr{F}$ and $D \cap\left(D^{c} \cup\{y\}\right)=\{y\} \in \gamma$, which is impossible. Therefore, for every $b>x_{0},\left\{x \in P \mid x_{0}<x<b\right\} \cap V$ is $\tau$-dense in $I \cap P$, for some open interval $I \subset R$. Let $\left\{J_{n}\right\}_{n=1}^{\infty}$ be a family of disjoint $\tau$-open subsets of $P$ whose union is the $\tau$-interior of the $\tau$-closure of $V \cap\left\{x \in P \mid x>x_{0}\right\}$.

For each positive integer $n$, there exists an $F \in \mathscr{F}$ such that $V \cap J_{n}=F \cap J_{n}$. [Suppose there exists some $n$ such that for each $F \in \mathscr{F}, V \cap J_{n} \neq F \cap J_{n}$. Since $\mathscr{F}$ is a maximal filter of $\tau$-dense subsets of $P$ and since $V \cap J_{n}$ is $\tau$-dense in $J_{n}$, this implies that there exists some $F_{1} \in \mathscr{F}$ such that $\left(V \cap J_{n}\right) \cap F_{1}$ is not $\tau$-dense in $J_{n}$. Hence, there exists some $\tau$-open set $L \subset J_{n}$ such that $L \cap F_{1} \cap$ $\left(V \cap J_{n}\right)=\phi$. If $x$ is a fixed element of $L \cap\left(V \cap J_{n}\right)$, then $\left(F_{1} \cup\{x\}\right) \in \mathscr{F}$ and $\left(F_{1} \cup\{x\}\right) \cap L \cap\left(V \cap J_{n}\right)=\{x\} \in \gamma$, which is impossible.]

Put $G=\left\{x_{0}\right\} \cup\left\{\bigcup_{n}^{\infty} J_{n}\right\} . G$ is an $N$-set. Also, $G \in \mathscr{C l}$. [For, if $G \notin \mathscr{L}, G \cap G_{1}$ is not an $N$-set for some $G_{1} \in \mathscr{M}$. This implies there exists some $x_{1} \in G \cap G_{1}$ and some $b>x_{1}$ such that $\left\{x \in P \mid x_{1}<x<b\right\} \cap$ $G \cap G_{1}$ does not contain a $\tau$-open set. It is clear that $x_{1}=x_{0}$. It follows as a consequence of how $G$ was constructed from $V$, that $C=V \cap\left\{x \in P \mid x_{0}<x<b\right\} \cap G_{1}$ is a $\tau$-nowhere dense subset of $P$. Also, $C \in \gamma . \quad C^{c} \in \mathscr{F}$ and if $y \in C$, then $\left(C^{c} \cup\{y\}\right) \cap C=\{y\} \in \gamma$. If $C$ is empty, then, since $\left\{x \in P \mid x_{0} \leqq x<b\right\} \in \mathscr{L}$, it follows that $V \cap$ $\left\{x \in P \mid x_{0} \leqq x<b\right\} \cap G_{1}=\left\{x_{0}\right\} \in \gamma$. A contradiction, therefore, $G \in \mathbb{C}$. $]$

Now, put $T=\left[\bigcup_{n=1}^{\infty}\left\{V \cap J_{n}\right\}\right] \cup\left\{\bigcup_{n=1}^{\infty} J_{n}\right\}^{c}$. For each $\tau$-open set $U \subset P, T$ is $\tau$-dense in $U$. $\mathscr{F}$ is a maximal filter of $\tau$-dense subsets of $P$. If $T \notin \mathscr{F}$, then there exists some $F^{\prime \prime} \in \mathscr{F}$ such that $T \cup F^{\prime \prime}$ is not $\tau$-dense in $P$. By the definition of $T$, this implies that $F^{\prime \prime} \cap$ $\left(V \cap J_{n}\right)$ is not dense in $J_{n}$ for some $n$. However, this is impossible since $V \cap J_{n}=F \cap J_{n}$, for some $F \in \mathscr{F}$, and $\mathscr{F}$ is a filter of $\tau$-dense subsets of $P$. Therefore $T \in \mathscr{F}$. Clearly $x_{0} \in G \cap T$ and $G \cap T \subset V$. Also, $G \cap T \in \sigma$, since $G \in \mathscr{C}$ and $T \in \mathscr{F}$. Therefore, $G \cap T \subset V$ is a $\sigma$-open neighborhood of $x_{0}$, which, since $x_{0}$ is an arbitrary element of $V$, contradicts the assumption that $V \notin \sigma$. Hence, $(P, \sigma)$ is maximal perfect and the lemma is established.

3. Example. We now give an example of a countable connected Hausdorff space $(X, \sigma)$ which is not contained in any maximal connected space. The space $(X, \sigma)$ is a modification of a countable connected Urysohn space which was constructed by P. Roy (see [3]). Let $\left\{E_{i}\right\}_{i=+\infty}^{-\infty}$ be a countable, disjoint collection of dense subsets 
of rational numbers indexed by the set of all integers. For each integer $n$, let $P_{n}=\left\{(x, n) \mid x \in E_{n}\right\}$. Let $w$ denote an ideal point. Let $X=\{w\} \cup\left\{\bigcup_{n=-\infty}^{+\infty} P_{n}\right\}$. We may consider $X$ as a collection of points in the plane lying on horizontal lines with integer ordinates together with the point $w$. We now construct a neighborhood system for the points of $X$.

(a) If $n$ is even, then put a maximal perfect topology $\sigma_{n}$ on $P_{n}$ exactly as described in $\S 2$.

(b) Let $n \neq 1$ be an odd integer and let $p=(x, n) \in P_{n}$. Then, for each positive integer $m$, let

$$
\begin{aligned}
U_{m}(p) & =\left\{(y, n+1) \in P_{n+1}|| x-y \mid\right. \\
& \left.<\frac{1}{m}\right\} \cup\left\{(y, n-1) \in P_{n-1}|| x-y \mid<\frac{1}{m}\right\} \cup\{p\} .
\end{aligned}
$$

(c) Let $n=1$ and $p=(x, n) \in P_{n}$. If $x \notin(\pi / 2, \pi)$, then, for each positive integer $m$, define $U_{m}(p)$ as in (b). If $\pi / 2<x<\pi$, then, for each positive integer $m$, let $U_{m}(p)=\left\{(y, n-1) \in P_{n-1}|| x-y \mid<\right.$ $1 / m\} \cup\{p\}$.

(d) If $p=w$, then, for each positive integer $m$, let $U_{m}(p)=$ $\{p\} \cup\{(x, n)|| n \mid \geqq 2 m\}$ be a neighborhood of $p$.

It is clear that the neighborhood system described above generates a topology $\sigma$ on $X . X$ is countable and it is easily seen that $(X, \sigma)$ is a Hausdorff space. We state the following lemmas (a slight modification of Lemmas 1, 2, and 3 of [3]) without proof. However, it is not difficult to verify that these lemmas also hold for the space $(X, \sigma)$.

Lemma 3. Suppose $p=(x, n)$, where $n$ is an even integer. Let $U$ be a basic open set containing $p$ and let $m$ be an integer.

(i) If $p \notin\{(x, n) \mid n=2$ and $\pi / 2<x<\pi\}$, then $\left(\mathrm{cl}_{o} U\right) \cap P_{m} \neq \phi$, if and only if $|m-n| \leqq 1$.

(ii) If $p=(x, 2)$, where $x \in(\pi / 2, \pi)$, then $\left(\operatorname{cl}_{\sigma} U\right) \cap P_{m} \neq \phi$, if and only if $m=2$ or 3 .

(iii) $w \notin \mathrm{cl}_{\sigma} U$.

Lemma 4. Suppose $p=(x, n)$, where $n$ is an odd integer. Let $U$ be a basic open set containing $p$ and let $m$ be an integer.

(i ) If $p \notin\{(x, n) \mid n=1$ or 3 and $\pi / 2<x<\pi\}$, then $\left(\mathrm{cl}_{\sigma} U\right) \cap$ $P_{m} \neq \phi$, if and only if, $|m-n| \leqq 2$.

(ii) If $p=(x, 1)$, where $x \in(\pi / 2, \pi)$, then $\left(\mathrm{cl}_{o} U\right) \cap P_{m} \neq \phi$, if and only if, $m=1,0$ or -1 .

(iii) If $p=(x, 3)$, where $x \in(\pi / 2, \pi)$, then $\left(\mathrm{cl}_{\sigma} U\right) \cap P_{m} \neq \phi$ if and only if, $m=2,3,4$ or 5 . 


\section{(iv) $w \notin \mathrm{cl}_{\sigma} U$.}

Lemma 5. Suppose $p=w, m$ is a positive integer greater than 1 , and $n$ is an integer. Then

(i ) $\operatorname{cl}_{\sigma} U_{m}(p) \supset P_{n}$, if $|n| \geqq 2 m-1$; and (ii) $\left\{\mathrm{cl}_{\sigma} U_{m}(p)\right\} \cap P_{n}=\phi$, if $|n|<2 m-1$.

LEMma $6 .(X, \sigma)$ is connected.

The proof of Lemma 6 is omitted since it is essentially the same as that given in [3].

Let $\gamma$ be any topology on $X$ such that $\gamma$ is larger than or equal to $\sigma$ and $(X, \gamma)$ is connected. We will now show that there exists a topology $\gamma^{\prime}$ on $X$ such that $\gamma^{\prime}$ is strictly larger than $\gamma$ and $\left(X, \gamma^{\prime}\right)$ is connected.

Let $I$ be any open interval in $R$ and, for each integer $n$, let $I_{n}=$ $\left\{(x, n) \in P_{n} \mid x \in I\right\}$. For each odd integer $n$, let $U\left(I_{n}\right)=\left\{x \in I \mid(x, n) \in I_{n}\right.$, $(x, n) \in \operatorname{cl}_{r} P_{n+1}$ and $\left.(x, n) \in \operatorname{cl}_{r} P_{n-1}\right\}$. Throughout, $I$ will denote an open interval of the real line with the usual topology. For each integer $n, I_{n}$ is a subset of $X$ and $U\left(I_{n}\right)$ is a subset of $I$ for each odd integer $n$.

Lemma 7. If $I$ is any open interval contained in $(\pi / 2, \pi)$, then $U\left(I_{n}\right)$ is dense in $I$ for all odd integers $n$, except for $n=1$.

Proof. It follows from condition (c) in the definition of the neighborhood base for the topology $\sigma$ on $X$, that for every open interval $I \subset(\pi / 2, \pi), U\left(I_{1}\right)=\phi$. Suppose there exists some interval $I \subset(\pi / 2, \pi)$ and an odd integer $n \neq 1$ such that $U\left(I_{n}\right)$ is not dense in $I$. We may assume without loss of generality that $I=(a, b)$, where $a$ and $b$ are irrationals, $U\left(I_{n}\right)=\phi$ and $n>1$. Put $H_{n}=$ $\left\{p \in I_{n} \mid p \notin \operatorname{cl}_{r} P_{n+1}\right\}$. Let $H=H_{n} \cup\left\{\bigcup_{k=2}^{n-1} I_{k}\right\}$. We will show that $H$ is both $\gamma$-open and $\gamma$-closed. Suppose $p \in H_{n}$, then $p \notin \mathrm{cl}_{\gamma} P_{n+1}$, so there exists a $\gamma$-open neighborhood, $V$, of $p$ which consists of $p$ and a subset of $I_{n-1}$. Hence $V \subset H$. Clearly, if $p \in I_{k}, k=2, \cdots, n-1$, there exists a $\sigma$-open set containing $p$ and contained in $\bigcup_{k=2}^{n-1} I_{k}$. Therefore, $H$ is $\gamma$-open.

If $p \in I_{n+1}$, then $p$ is not a $\sigma$-limit point of $H$ and, since $\sigma \subset \tau$, it follows that $p \notin \mathrm{cl}_{r} H$. If $p \in I_{n}$ and $p \notin H$, then $p$ is a $\gamma$-limit point of $I_{n+1}$ and, since $U\left(I_{n}\right)=\phi, p$ must have a $\gamma$-open neighborhood which consists of $p$ and a subset of $I_{n+1}$. Hence $p \notin \mathrm{cl}_{\gamma} H$. It follows from the construction of $\sigma$, that if $p \in P_{1}$ or $p \in P_{0}$, then $p \notin \mathrm{cl}_{r} H$. Also, if $p \in P_{k}$, for $k=2, \cdots, n-1$, and $p \notin H$, there exists a $\sigma$-open set containing $p$ which does not meet $H$. Therefore, $H$ is $\gamma$-closed. 
This contradicts the assumption that $(X, \gamma)$ is connected and the lemma is established.

We now make use of the preceding lemma to put a topology $\gamma^{\prime}$ on $X$ such that $\gamma^{\prime}$ is strictly larger than $\gamma$. Let $J$ be a fixed open interval contained in $(\pi / 2, \pi)$. It follows from Lemma 7 that $U\left(J_{n}\right)$ is dense in $J$ for all odd integers, except for $n=1$. Let $y_{0}$ be a fixed element of $U\left(J_{3}\right)$ and put $y=\left(y_{0}, 3\right)$. Let $V$ be an open neighborhood of $y$ in $\gamma$. It follows that $V \cap P_{4}$ and $V \cap P_{2}$ are both nonempty. Put $G=\{y\} \cup\left\{V \cap P_{2}\right\}$. Clearly $G \notin \gamma$. Put $\gamma^{\prime}=\gamma \vee G$. We will show (Theorem 1$)$ that $\left(X, \gamma^{\prime}\right)$ is connected. Throughout the remainder of this note, $G, \gamma^{\prime}, J$, and $y$ will be reserved for the roles assigned to them in this paragraph.

LEMMA 8. If $n$ is an integer and $V \subset P_{2 n}$, where $V \in \gamma^{\prime}$, then there exists some interval $I \subset R$ such that $\{x \mid(x, 2 n) \in V\}$ is dense in $I$.

Proof. Suppose not. Then there exists some integer $n$ and a $\gamma^{\prime}$-open set $V \subset P_{2 n}$ such that $\{x \mid(x, 2 n) \in V\}$ is not dense in any subinterval of $R$. Since $P_{2 n} \in \sigma$ and $\left(P_{2 n}, \sigma \mid P_{2 n}\right)$ is maximal perfect, it follows that $V \in \sigma$ [otherwise, some point of $V$ would be an isolated point of $\left.\gamma^{\prime}\right]$. Put $F=\left\{(x, 2 n) \in P_{2 n} \mid(x, 2 n) \notin V\right\}$. Since $\{x \mid(x, 2 n) \in V\}$ is not dense in any subinterval of $R$, it follows from the definition of the maximal filter $\mathscr{F}$ on $P_{2 n}$ that $F \in \mathscr{F}$. If $p \in V$, then $F \cup\{p\} \in \mathscr{F}$ and $(F \cup\{p\}) \in \sigma$. Therefore, $(F \cup\{p\}) \cap V=\{p\}$ and $\{p\} \in \sigma$. This contradiction establishes the lemma.

Lemma 9. Suppose $n$ is an integer and $V$ and $U$ are open subsets of $P_{2 n}$. If $\{x \mid(x, 2 n) \in V\}$ and $\{x \mid(x, 2 n) \in U\}$ are both dense in some open interval $I$, then $V \cap U \neq \phi$.

Proof. Suppose $V \cap U=\phi$. Since $\{x \mid(x, 2 n) \in V\}$ and $\{x \mid(x, 2 n) \in U\}$ are both dense in $I$, we may assume that $V=I_{2 n} \cap F_{1}$ and $U=I_{2 n} \cap F_{2}$, where $F_{1}, F_{2} \in \mathscr{F}$ and $\mathscr{F}$ is the maximal filter of dense sets used to construct the neighborhood system of $P_{2 n}$. If $p \in V$, then $F_{2} \cup$ $\{p\} \in \mathscr{F}$. Hence, $U \cup\{p\}=\left(F_{2} \cap\{p\}\right) \cap I_{2 n}$ is a $\sigma$-open subset of $P_{2 n}$. However, $(U \cup\{p\}) \cap V=\{p\}$ and $\{p\} \in \sigma$. This contradiction establishes the lemma.

LEMMA 10. Let $U$ be a $\gamma^{\prime}$-open neighborhood of $w$. If $I$ is any open interval contained in $J$, then there exists an integer $n \geqq 2$ such that $I_{n} \cap U \neq \phi$.

Proof. Suppose for some $\gamma^{\prime}$-open set $U$ containing $w$, there exists 
an interval $I \subset J, I=(a, b)$, where $a$ and $b$ are irrational numbers, such that $I_{n} \cap U=\phi$, for each integer $n \geqq 2$. Since every $\gamma^{\prime}$-open set containing $w$ contains a $\gamma$-open neighborhood of $w$, we may assume that $U \in \gamma$. Put $H=\bigcup_{n=2}^{+\infty} I_{n}$. It follows that $H$ is $\gamma$-open. $H$ is also $\gamma$-closed. For, if $p \in I_{1}, p$ has a $\sigma$-open neighborhood which does not meet $H$, and by assumption, $w$ has a $\gamma$-open neighborhood which does not meet $H$. This contradicts the assumption that $(X, \gamma)$ is connected and the lemma follows.

Corollary. Let $I \subset J$. If $U$ is any $\gamma^{\prime}$-open set containing $w$, then there exists a infinite set of positive integers, $N$, such that $I_{n} \cap U \neq \phi$, for each $n \in N$.

Lemma 11. Let $n$ be an integer such that $n \geqq 1$. Let $V$ be an open subset of $P_{2 n}$ such that $\{x \mid(x, 2 n) \in V\}$ is dense in an open interval $I \subset J$. If $V \subset K$, where $K$ is an open and closed set in $\left(X, \gamma^{\prime}\right)$, then $I_{k} \subset K$, for all $k>2 n$.

Proof. First we will show that $I_{2 n+1}$ and $I_{2 n+2}$ are both contained in $K . U\left(I_{2 n+1}\right)=\left\{x \in I \mid p=(x, 2 n+1) \in I_{2 n+1}\right.$ and $p$ is a $\gamma$-limit point of both $I_{2 n}$ and $\left.I_{2 n+2}\right\}$. It follows from Lemma 7 and the choice of $J$, that, since $I \subset J, U\left(I_{2 n+1}\right)$ is dense in $I$. Let $p=(x, 2 n+1)$, where $x \in U\left(I_{2 n+1}\right)$. Let $U$ be a $\gamma^{\prime}$-open set containing $p$ (we may, without loss of generality, assume that $U \in \gamma)$. Since $U \cap I_{2 n} \neq \phi$, it follows from Lemma 8 that there exists some open interval $I^{\prime} \subset I$ such that $\{x \mid(x, 2 n) \in U\}$ is dense in $I^{\prime}$. Also, $\{x \mid(x, 2 n) \in V\}$ is dense in $I^{\prime}$. Therefore, by Lemma 9, $V \cap U \neq \phi$. Since $V \subset K, U \cap K \neq \phi$ and $p$ is therefore a limit point of $K$. Since $K$ is $\gamma^{\prime}$-closed, $p \in K$. Therefore, $\left\{p \mid p=(x, 2 n+1)\right.$ and $\left.x \in U\left(I_{2 n+1}\right)\right\} \subset K$.

Put $Q=\left\{x \in I \mid q=(x, 2 n+2) \in I_{2 n+2}\right.$ and $\left.q \in K\right\}$. Since $U\left(I_{2 n+1}\right)$ is dense in $I, Q$ is also dense in $I$. Let $z \in I_{2 n+2}$. It will be shown that $z$ is a $\gamma^{\prime}$-limit point of $K$. Let $H$ be a $\gamma^{\prime}$-open set containing $z$. We may assume that $H \subset P_{2 n+2}$. Again, by Lemma 8, there exists an interval $I^{\prime} \subset I$ such that $\{x \mid(x, 2 n+2) \in H\}$ is dense in $I^{\prime}$. Since $Q$ is dense in $I, K \cap I^{\prime}{ }_{2 n+2} \neq \phi$. This implies that $K \cap I^{\prime}{ }_{2 n+2} \in \gamma^{\prime}$ and, by Lemma 8 , we may assume without loss of generality, that $K \cap I^{\prime}{ }_{2 n+2}$ is dense in $I^{\prime}{ }_{2 n+2}$. By Lemma 9, $\left(K \cap I^{\prime}{ }_{2 n+2}\right) \cap H \neq \phi$. Therefore, $z$ is a $\gamma^{\prime}$-limit point of $K$ and since $K$ is $\gamma^{\prime}$-closed, $z \in K$. Hence, $I_{2 n+2} \subset K$.

Now, suppose there exists some $p \in I_{2 n+1}$ such that $p \notin K$. Then there exists a $\gamma^{\prime}$-open set $L$ containing $p$ such that $L \cap I_{2 n+2}=\phi$ and $L \cap V=\phi$. Using Lemmas 8 and 9 , this can be shown to be impossible. Hence $I_{2 n+1} \subset K$. It can now be shown by induction that, for all $k>2 n, I_{k} \subset K$.

Theorem 1. $\left(X, \gamma^{\prime}\right)$ is connected. 
Proof. Let $y$ be that element of $J_{3}$, where $J \subset(\pi / 2, \pi)$, such that $\gamma^{\prime}=\gamma \vee G, y \in G$ and $G \notin \gamma$. Suppose there exists a set $K \subset X$ such that $K$ is both open and closed in $\left(X, \gamma^{\prime}\right)$. Assume $y \notin K$. This implies that $K \in \gamma$. Since $(X, \gamma)$ is connected, $K$ is not closed in $(X, \gamma)$, Therefore, if $U$ is a $\gamma$-open neighborhood of $y, U \cap K \neq \phi$. Hence, $U \cap\left(P_{2} \cap K\right) \neq \phi$ or $U \cap\left(P_{4} \cap K\right) \neq \phi$. Since $G=\{y\} \cup\left(V \cap P_{2}\right)$, where $V$ is a fixed $\gamma$-open neighborhood of $y$, and $y$ is not a $\gamma^{\prime}$-limit point of $K$, it follows that $U \cap\left(P_{4} \cap K\right) \neq \phi$. By Lemma 8, there exists a $\gamma^{\prime}$-open set $U_{4} \subset U \cap\left(P_{4} \cap K\right)$ such that $\left\{x \mid(x, 4) \in U_{4}\right\}$ is dense in an open interval $I \subset J$. Lemma 11 implies, that, for all $k>4, I_{k} \subset K$. Then, by the corollary following Lemma 10, $w \in K$.

Since $y \notin K, y \in K^{\circ}$ and $K^{c}$ is also open and closed in $\left(X, \gamma^{\prime}\right)$. Let $H$ be a $\gamma^{\prime}$-open neighborhood of $y$ such that $H \subset K^{\circ}$. Then $H \cap P_{2} \neq \phi$. This implies, by Lemma 8 , that there exists a $\gamma^{\prime}$-open set $H_{2} \subset H \cap$ $P_{2} \cap K^{c}$ such that $\left\{x \mid(x, 2) \in H_{2}\right\}$ is dense in some open interval $I^{\prime} \subset J$. Again, it follows from Lemmas 10 and 11 that $w \in K^{\circ}$. This contradicts the fact that $K \cap K^{c}=\phi$. Therefore, $\left(X, \gamma^{\prime}\right)$ is connected.

It is now and immediate consequence of the following theorem $(X, \sigma)$ cannot be embedded into any maximal connected space.

THEOREM 2. (See [2]). Every connected subspace of a maximal connected space is maximal connected.

REMARK 2. If for each $n, E_{n}$ is an uncountable dense subset of the irrationals, it follows that one can then construct an uncountable connected Hausdorff space which cannot be embedded into any maximal connected space.

Question. What connected Hausdorff spaces have the property that they cannot be embedded into a maximal connected space?

Acknowledgements. The author is grateful to the referee for his suggestions.

\section{REFERENCES}

1. N. Bourbaki, General Topology, Part 1, Addison-Wesley, Reading, 1966.

2. J. A. Guthrie and H. F. Stone, Subspaces of maximally connected spaces, Notices Amer. Math. Soc., 18 (1971), 672.

3. P. Roy, A countable connected Urysohn space with a dispersion point, Duke Math. J., 33 (1966), 331-333.

4. J. P. Thomas, Maximal connected topologies, Austr. Math. Soc. J., 8 (1968), 700-705.

Received November 13, 1972 and in revised form April 30, 1973. This research was partly supported by the National Research Council of Canada, Grant No. A8016. 


\section{PACIFIC JOURNAL OF MATHEMATICS}

\section{EDITORS}

RICHARD ARENS (Managing Editor)

University of California

Los Angeles, California 90024

R. A. BeAumont

University of Washington

Seattle, Washington 98105
J. DugundJI*

Department of Mathematics

University of Southern California

Los Angeles, California 90007

D. Gilbarg and J. Milgram

Stanford University

Stanford, California 94305

\section{ASSOCIATE EDITORS}

E. F. BECKENBACH

B. H. NEUMANN

F. WOLF

K. YoSHIDA

\section{SUPPORTING INSTITUTIONS}

UNIVERSITY OF BRITISH COLUMBIA
CALIFORNIA INSTITUTE OF TECHNOLOGY
UNIVERSITY OF CALIFORNIA
MONTANA STATE UNIVERSITY
UNIVERSITY OF NEVADA
NEW MEXICO STATE UNIVERSITY
OREGON STATE UNIVERSITY
UNIVERSITY OF OREGON
OSAKA UNIVERSITY

UNIVERSITY OF BRITISH COLUMBIA CALIFORNIA INSTITUTE OF TECHNOLOGY UNIVERSITY OF CALIFORNIA MONTANA STATE UNIVERSITY NEW MEXICO STATE UNIVERSITY UNIVERSITY OF OREGON OSAKA UNIVERSITY
UNIVERSITY OF SOUTHERN CALIFORNIA STANFORD UNIVERSITY UNIVERSITY OF TOKYO UNIVERSITY OF UTAH WASHINGTON STATE UNIVERSITY UNIVERSITY OF WASHINGTON AMERICAN MATHEMATICAL SOCIETY NAVAL WEAPONS CENTER

* C. R. DePrima California Institute of Technology, Pasadena, CA 91109, will replace J. Dugundji until August 1974. 


\section{Pacific Journal of Mathematics}

\section{Vol. 51, No. $1 \quad$ November, 1974}

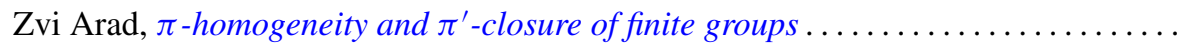

Ivan Baggs, A connected Hausdorff space which is not contained in a maximal

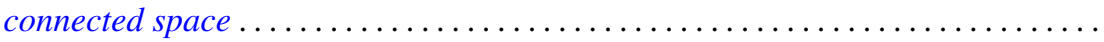

Eric Bedford, The Dirichlet problem for some overdetermined systems on the unit ball in $C^{n}$

R. H. Bing, Woodrow Wilson Bledsoe and R. Daniel Mauldin, Sets generated by

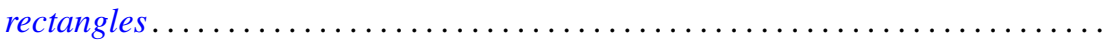

Carlo Cecchini and Alessandro Figà-Talamanca, Projections of uniqueness for

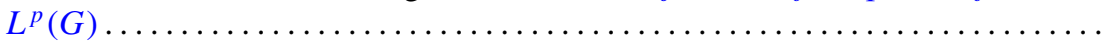

Gokulananda Das and Ram N. Mohapatra, The non absolute Nörlund summability of Fourier series .

Frank Rimi DeMeyer, On separable polynomials over a commutative ring ........ Richard Detmer, Sets which are tame in arcs in $E^{3} \ldots \ldots \ldots \ldots \ldots \ldots \ldots \ldots$

William Erb Dietrich, Ideals in convolution algebras on Abelian groups ..........

Bryce L. Elkins, A Galois theory for linear topological rings .................

William Alan Feldman, A characterization of the topology of compact convergence on $C(X)$.

Hillel Halkin Gershenson, A problem in compact Lie groups and framed cobordism

Samuel R. Gordon, Associators in simple algebras.

Marvin J. Greenberg, Strictly local solutions of Diophantine equations

Jon Craig Helton, Product integrals and inverses in normed rings . . . . . . . . . . . .

Domingo Antonio Herrero, Inner functions under uniform topology . . .

Jerry Alan Johnson, Lipschitz spaces .

Marvin Stanford Keener, Oscillatory solutions and multi-point boundary value

functions for certain nth-order linear ordinary differential equations.

John Cronan Kieffer, A simple proof of the Moy-Perez generalization of the

Shannon-McMillan theorem .......................

Joong Ho Kim, Power invariant rings

Gangaram S. Ladde and V. Lakshmikantham, On flow-invariant sets .

Roger T. Lewis, Oscillation and nonoscillation criteria for some self-adjoint even

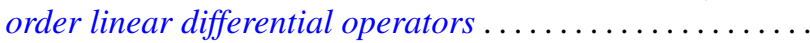

Jürg Thomas Marti, On the existence of support points of solid convex sets ..

John Rowlay Martin, Determining knot types from diagrams of knots . .

James Jerome Metzger, Local ideals in a topological algebra of entire functions

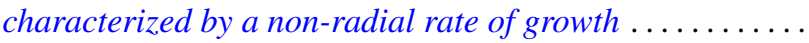

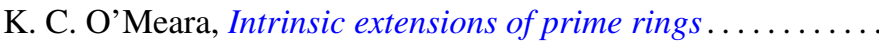

Stanley Poreda, A note on the continuity of best polynomial approximations ..

Robert John Sacker, Asymptotic approach to periodic orbits and local prolongations

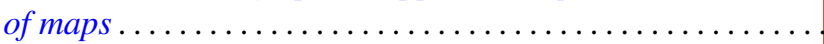

Eric Peter Smith, The Garabedian function of an arbitrary compact set . .

Arne Stray, Pointwise bounded approximation by functions satisfying a side condition

John St. Clair Werth, Jr., Maximal pure subgroups of torsion complete abelian

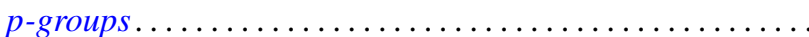

